\title{
La Prevención del VIH/SIDA en la formación universitaria de los futuros educadores
}

Carmen María Aránzazu Cejudo Cortés

Universidad de Huelva (España)

Celia Corchuelo Fernández

Universidad de Huelva (España) 



\title{
La Prevención del VIH/SIDA en la formación universitaria de los futuros educadores
}

\section{The prevention of HIV/AIDS in the university training of teachers and social educators}

\author{
Carmen María Aránzazu Cejudo Cortés \\ Universidad de Huelva (España) \\ carmen.cejudo@dedu.uhu.es
}

\author{
Celia Corchuelo Fernández \\ Universidad de Huelva (España) \\ celia.cochuelo@dedu.uhu.es
}

Fecha de recepción: 9 de diciembre de 2017

Fecha de aceptación: 26 de abril de 2019

\section{Resumen}

En esta investigación se presentan algunos de los resultados sobre los conocimientos de los estudiantes de titulaciones educativas de la Universidad de Huelva sobre el VIH/SIDA, referidos al grado y calidad del conocimiento que tienen sobre esta enfermedad. Gracias a ellos, hemos podido explorar e identificar las repercusiones derivadas de la falta o insuficiencia de información veraz y, proponer acciones educativas en el ámbito universitario, entendido como agente promotor de salud. Mediante un cuestionario aplicado a una muestra de más de seiscientos sujetos, hemos logrado averiguar que, la mitad de ellos obtienen un resultado insuficiente en la prueba de conocimiento, la desinformación de cuestiones capitales -como, las vías de transmisión del VIH- aún persisten. En definitiva, se sugiere la necesidad de elaborar y desarrollar propuestas formativas que mejoren las competencias en esta materia de estos estudiantes, que además serán futuros educadores en ámbitos escolares y sociales.

Palabras clave: Promoción de la salud; Docentes; VIH/SIDA; Universidad.

\footnotetext{
Abstract

This research presents some of the results on University of Huelva Education Degrees students' knowledge towards the HIV/ AIDS, regarding the degree and quality of their knowledge of the disease. Thank to them, we have been able to explore and identify the effects of the absence or lack of veracious information
} 
and, consequently, to propose educational actions in the University sphere, which is viewed as a health promotion agent. By means of a questionnaire applied to a sample of over six hundred subjects we managed to discover that, half of them have an insufficient result in the knowledge test, the disinformation of fundamental issues - like ways of transmission of the HIV - remains. In short, our analysis evokes the need to elaborate and develop educational proposals, which would improve the students' competencies in this matter, these students being future education professionals in the school and social spheres.

Key words: Health promotion; Education professionals; HIV/AIDS; University.

\section{INTRODUCGIÓN}

En los últimos años ha ganado en relevancia el estudio y desarrollo de la universidad como espacio para la promoción de hábitos saludables. Una promoción que debe partir desde distintos ámbitos de la sociedad, tanto por parte de la familia, de las instituciones, así como por los profesionales de la educación. Estos últimos, deben poseer una serie de requisitos en formación de hábitos de vida saludable. De esta manera, las instituciones universitarias deben inculcar a los futuros discentes todos los aspectos que sobre esta materia son imprescindibles. Los jóvenes de hoy en día tienen la necesidad de relacionarse saludablemente y, por consiguiente, formarse en salud desde un punto de vista distinto al normativo.

Burkhart (2009) denomina a las normas descriptivas como aquellas que indican "lo que realmente hace la gente en un contexto determinado, independiente de la aprobación". Estas se encuentran, por lo general, implícitas y establecen la manera de actuar en la sociedad y la forma de adaptarnos a determinados grupos en los que deseamos incluirnos. Tanto es así que, un número considerable de jóvenes actualmente, no tienden a llevar un control del riesgo en las prácticas sexuales, siendo la población más afectada en las nuevas infecciones por VIH/SIDA, tal como alertan diferentes instituciones epidemiológicas como el Programa de las Naciones Unidas sobre el VIH/SIDA (ONUSIDA), la Fundación para la Investigación y Prevención del Sida en España (FIPSE) o el Plan Andaluz frente al VIH/SIDA.

Por eso, a raíz de esos informes, se manifiesta la necesidad de continuar desarrollando políticas de prevención y estrategias de intervención socio-educativa en los jóvenes de hoy en día. Algunos de los datos que refleja el Informe de ONUSIDA (2018), indica que 36.9 millones de personas viven con VIH en todo el mundo, unos dos millones más que en 2009 gracias a que han podido acceder más personas al tratamiento antirretroviral. La caída del número de nuevas infecciones (1.8 millones en 2017) es, no obstante, insuficiente y está aún lejos de la meta que se marcó en el programa "Getting to Zero: 2011-2015 Strategy" en 2010.

Desde las instituciones escolares se deben desarrollar valores relacionados con la convivencia y la tolerancia hacia la diversidad mediante el aprendizaje cooperativo, la empatía y el desarrollo de una serie recursos personales y sociales (Cava y Musitu, 


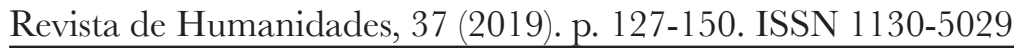

2002; Martxueta et al., 2014), y en el caso que nos atañe, es primordial por la formación de los futuros educadores. El Plan Multisectorial frente a la infección por VIH y el Sida en España (2008-2012) del Ministerio de Sanidad y Consumo (2008) señala que, durante muchos años, nuestro país ha tenido las tasas más altas de infección de Europa. Este informe indicó que entre 120.000 y 150.000 ciudadanos españoles están infectados por el VIH, y estima que más de una cuarta parte de los infectados aún ignora que lo está.

Multitud de estudios intentan informar sobre los progresos llevados a cabo sobre el VIH/SIDA, sus limitaciones y desafíos en la promoción de hábitos saludables, como en la educación para la salud en la escuela (Salvador, 2008; Meneses et al., 2009; Aramendi et al., 2014; Vega et al., 2015). La Estrategia de la UNESCO sobre el VIH/SIDA (UNESCO, 2012), recoge esa necesidad con una serie de metas para la prevención y anima a los centros escolares a que propicien un contexto generador de salud.

Una promoción de hábitos saludables, que, como vía de prevención de enfermedades y adicciones, se ha visto potenciada a lo largo de los últimos años y ha encontrado su marco ideal en las universidades. Esta promoción es llevada a cabo por las escuelas y las universidades, a través de una serie de actuaciones que en la mayoría de los casos, la imparten profesionales sanitarios. En resumidas cuentas, estas actuaciones aisladas y no perdurables en el tiempo, calan poco al no estar incluidas en el currículo de las diversas etapas educativas y, por tanto, desemboca en el incumplimiento de una verdadera escuela promotora de salud (OMS, 2010; Vega et al, 2015). Sin embargo, González (2009) afirma que la tendencia está cambiando, ya que la promoción de la salud -sobre todo en el ámbito universitario- están teniendo un creciente reconocimiento e importancia tanto a nivel mundial como nacional. La universidad, por consiguiente, es un contexto idóneo para la generación de este movimiento, pues es en la universidad donde se preparan durante años cruciales de su vida, los profesionales que después desempeñarán un papel relevante en su terreno. La universidad es, sin duda, el espacio físico y temporal perfecto para promover buenas prácticas de salud, que, además, producirán grandes beneficios en el contexto social presente y futuro.

\section{LA INVESTIGAGIÓN SOBRE PROMOGIÓN DE LA SALUD Y PREVENCIÓN DEL VIH EN EL ALUMNADO UNIVERSITARIO}

La falta de conocimiento que expresa el profesorado en la transmisión y fomento de los hábitos saludables es a la conclusión que llegan numerosos estudios. Se desconoce aspectos básicos de esa temática y, en consecuencia, la información que ofrecen a sus alumnos es escasa, parcial y ocasional (Palomares et al, 2014).

Un número considerable de investigaciones sobre la promoción de la salud y la prevención del VIH en el alumnado universitario sirven de base para justificar el 
argumento de análisis de este artículo, fundamentándose en la necesidad de integrar la educación sobre el VIH desde las etapas tempranas de la escolarización.

En contraposición a la formación tradicional que se hace en los centros educativos actualmente, Schenker y Nyirenda (2002) proponen seis perspectivas metodológicas: 1. Capacitar y comprometer a los profesionales de la educación; 2. Fomentar alianzas dentro de la escuela y de la comunidad. 3. Uso de métodos educativos no convencionales. 4. Presentación de una comunicación abierta. 5. Realización de sesiones innovadoras de enseñanza. 6. Enfoques de género específicos. Es decir, evitar sesiones formativas aisladas y a una edad tardía, a favor de una formación específica del docente, una implicación de toda la comunidad y metodologías más innovadoras que propicien el diálogo abierto sin tabúes en este ámbito.

Una de las líneas de investigación que más ocupa a los expertos en esta materia, es la efectividad o no de la campañas cuyo objetivo en la prevención de la infección del VIH. Las conclusiones esclarecen las dificultades existentes para poder realizar la prueba de detección del VIH en poblaciones con mayor riesgo como pueden ser lo jóvenes (De Wit y Adam, 2008, Deblonde et al., 2010, Mahajan et al., 2008, Prost et al., 2008, Morales et al., 2016). Mientras que las cifras de contagio por transfusiones de sangre, transmisión maternofilial o consumo de drogas por vía intravenosa han descendido de manera notable, el aumento de los casos por conductas sexuales de riesgo -particularmente entre la población joven- ha ganado terreno y se ha convertido en la principal vía de transmisión del virus. Y no es que haya desconocimiento acerca de los métodos de prevención del VIH entre los estudiantes universitarios, sino que, durante los últimos años, estos parecen haberse relajado al amparo de creencias erróneas como "a mí no me va a pasar" o "esa enfermedad ya no existe o está bajo control". En la disertación de Cassá at al. (2009), se resumieron algunas de las consecuencias de esta "relajación", como las referentes a no usar el preservativo (Osorio et al., 2017) o no hacerlo sistemáticamente, o ser reticentes a realizarse la prueba de detección de anticuerpos. Exploraron las causas y llegaron a la conclusión de que el alumnado universitario, en general, suele disponer de suficiente información sobre métodos de prevención de enfermedades de transmisión sexual. Aseveran que hay otras causas que propician llevar a cabo conductas sexuales de riesgo, como es el consumo de alcohol o drogas o, simplemente, el atractivo de hacer algo peligroso.

De la investigación de Hoyos et al. (2011), sorprende el bajo número de positivos obtenidos en la prueba de detección del VIH, si se tiene en cuenta la frecuencia documentada de no utilización del preservativo. Por ello concluye que, los campus universitarios no constituyen un entorno de riesgo para la transmisión del VIH y estos programas de prueba de detección rápida deberían trasladarse a localizaciones 


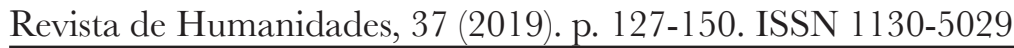

más vulnerables. De cualquier modo, la experiencia fue un paso adelante en la difusión de información preventiva y asesoramiento de educación para la salud ante reiteradas conductas de riesgo en la población estudiantil.

Muy esclarecedor y relevantes para el tema que nos ocupa es el estudio de Giménez et al. (2009). La investigación ratificó que las estrategias más eficaces fueron las charlas informativas, el juego de roles y la visualización de imágenes en formato audiovisual. Las charlas informativas incrementaron estadísticamente la predisposición a someterse a la prueba. En las actividades prácticas, como la de simular la colocación de un preservativo, ayudaron a romper tabúes y a aumentar la conciencia preventiva. Y en la experiencia audiovisual, como era de esperar, tuvo mayor incidencia a la hora de preguntar a la pareja acerca de la prueba, motivado por el miedo suscitado por determinadas imágenes.

El propósito de Fernández et al. (2006) fue el de verificar si una intervención pedagógicafundamentada enunametodología activa, queincluyeeltrabajocooperativo y el fomento de la discusión, era capaz de modificar en sentido positivo la actitud de los estudiantes ante el VIH/SIDA. En primer lugar, se les debe sensibilizar como estudiantes universitarios, para que afronten esa etapa de la vida con responsabilidad y competencia en la toma de decisiones; en segundo lugar, se les forma como profesionales, lo que, por su propia especialidad, les convierte en referentes para sus compañeros y para la comunidad, adoptando el rol de promotores de la salud.

La investigación de Tello (2009) trató de lanzar un número de propuestas con el objetivo de promover y fortalecer en los estudiantes universitarios, aquellos conocimientos y actitudes que les permitiera incrementar habilidades de autocuidado para alcanzar estilos de vida saludable. Pérez (2013) se centró en los conocimientos, actitudes y prácticas de riesgo del alumnado y el profesorado de un centro universitario del cual obtuvo numerosos y valiosos resultados.

El trabajo de Rojas-Murcia et al. (2014) se centró también en estudiantes universitarios Se concluyó con la necesidad de llevar a cabo programas de prevención para disminuir la ilusión invulnerabilidad de un buen porcentaje de jóvenes universitarios, la percepción de control y la aparición de prejuicios ante las personas con VIH/SIDA. En relación a este último aspecto -mejorar la actitud ante las personas con la enfermedad- se refiere el estudio de Bilbao et al. (2017) que tiene como participantes estudiantes de medicina.

En todo caso, un profesorado universitario sólidamente capacitado será capaz de promover una educación para la salud que cale en los estudiantes y logre transformar sus comportamientos de riesgo en hábitos saludables (Domínguez, Beltrán y Horta, 2017; Morán y Tarajano, 2017). Por ello, es sumamente importante que las universidades, en trabajo conjunto con los organismos de salud, se preocupen por llevar a cabo un control más exhaustivo de los conocimientos y actitudes de 
la población estudiantil a fin de determinar qué acciones sería necesario ejecutar para intensificar la prevención y evitar posibles contagios (Meza-León et al. 2017; Salamanca y Romero González, 2017). Como se ha podido observar, el saber conocer los hábitos del alumnado universitario, tanto en lo referente a sus prácticas sexuales como a las adicciones o a las actividades de tiempo libre en general, puede ser de gran ayuda a la hora de prever el riesgo y organizar campañas de educación para la salud.

\section{OBJETIVOS Y MÉTODO DE NUESTRA INVESTIGACIÓN}

La investigación que aquí se presenta, se ha centrado exclusivamente en los estudiantes universitarios de titulaciones educativas (principalmente Magisterio, Educación Social y diversos másteres) con una cuantiosa muestra de alumnado de la Universidad de Huelva. El objetivo general de la misma ha sido conocer las ideas, representaciones y actitudes de los estudiantes de estas titulaciones sobre el VIH/SIDA, a fin de identificar el grado y calidad de su información y poder así, planificar actuaciones educativas apropiadas en el ámbito universitario. En concreto la investigación pretende:

a. Valorar el nivel de conocimientos sobre VIH/SIDA de los estudiantes en relación a las cuestiones más relevantes.

b. Registrar las percepciones, imágenes y representaciones que los estudiantes tienen sobre la enfermedad.

c. Conocer las actitudes discriminatorias y/o de encubrimiento, que puedan producirse, en las relaciones de cualquier tipo con personas con VIH.

d. Y, por último, ofrecer evidencia científica útil para promover políticas sociales preventivas y programas de sensibilización e información sobre el VIH/SIDA en el medio universitario, entendido como entorno promotor de salud.

Hemos establecido una muestra de 613 sujetos, 122 más de los necesarios (491) para conseguir la suficiente representatividad para una población de 2076 estudiantes de la Facultad de Ciencias de Educación de la Universidad de Huelva, con un margen de error $(e)= \pm 0,04$ y con un nivel de confianza $(z=1,96)$ del $95 \%$. El modelo de muestreo utilizado ha sido el estratificado (por edad) y por cuotas o rutas (por cursos). Cada estrato o tramo de edad está representado con una frecuencia superior a $110 \mathrm{e}$ inferior a 170 sujetos, a fin de garantizar que quedaran debidamente representados todos los intervalos definidos. La frecuencia de estudiantes va decreciendo a medida que el intervalo de edad crece. 


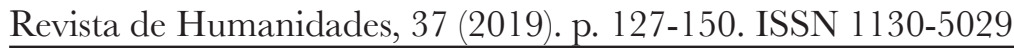

Tabla 1. Edad de los sujetos de la muestra

\begin{tabular}{llrrrr}
\hline & & Frecuencia & Porcentaje & Porcent. válido & \multicolumn{1}{c}{$\begin{array}{c}\text { Porcent. } \\
\text { acumulado }\end{array}$} \\
\hline \multirow{2}{*}{ Válidos } & 21 años o menos & 169 & 27,6 & 28,7 & 28,7 \\
\cline { 2 - 6 } & Entre 21 hasta 23 & 163 & 26,6 & 27,7 & 56,4 \\
\cline { 2 - 6 } & Entre 23 hasta 25 & 138 & 22,5 & 23,4 & 79,8 \\
\cline { 2 - 6 } & Mayor de 25 años & 119 & 19,4 & 20,2 & 100,0 \\
\cline { 2 - 6 } & Total & 589 & 96,1 & 100,0 & \\
\hline Perdidos & Sistema & 24 & 3,9 & & \\
\hline Total & & $\mathbf{6 1 3}$ & $\mathbf{1 0 0 , 0}$ & & \\
\hline
\end{tabular}

La media de edad de los encuestados es de 24 años de edad. Estamos ante una muestra valiosa para el objeto de este estudio, puesto que no sólo se intenta conocer cómo los futuros maestros, profesores y educadores se enfrentan a una enfermedad tan estigmatizada, sino que además obtenemos un importante registro de datos sobre conocimientos, percepciones y actitudes de uno de los grupos más castigados por la epidemia del VIH en los países desarrollados, los jóvenes.

Tabla 2. Media de edad, rango y desviación típica
\begin{tabular}{ll}
\hline \multicolumn{2}{l}{ Estadísticos } \\
\hline Edad \\
\hline $\mathrm{N} \quad$ \\
\cline { 2 - 2 } \\
\cline { 2 - 2 } Media & Perdidos \\
\hline Desv. típ. & 602 \\
\hline Rango & 11 \\
\hline
\end{tabular}

Con respecto al sexo, el número de mujeres en la muestra es considerablemente superior al de hombres $80 \%$ de alumnas mujeres, correspondiente a 496 personas de la muestra- dato previsible por la feminización de las titulaciones educativas. Es, además, apreciablemente mayor el número de sujetos de titulaciones de grado que de posgrado, por la desproporción también de sus respectivos volúmenes.

Tabla 3. Sexo de los participantes en el cuestionario

\begin{tabular}{llll}
\hline & & Frecuencia & Porcentaje \\
\hline \multirow{3}{*}{ Válidos } & 0 & 6 & 1,0 \\
\cline { 2 - 4 } & Mujer & 496 & 80,9 \\
\cline { 2 - 4 } & Hombre & 111 & 18,1 \\
\cline { 2 - 4 } & Total & 613 & 100,0 \\
\hline
\end{tabular}


La Prevención del VIH/SIDA... - C. M.A.Cejudo y G.Corchuelo

Tabla 4. Sexo de los participantes en el cuestionario

\begin{tabular}{lcccc}
\hline Titulación & Mujeres & Hombres & Frecuencia & Porcentaje \\
\hline Educación Social & 166 & 27 & 193 & 31,5 \\
\hline Educación Primaria & 56 & 31 & 87 & 14,2 \\
\hline Educación Infantil & 113 & 0 & 113 & 18,4 \\
\hline $\begin{array}{l}\text { Ciencias de la Actividad Física y } \\
\text { del Deporte }\end{array}$ & 5 & 21 & 26 & 4,2 \\
\hline Máster Educación Especial & 21 & 1 & 22 & 3,6 \\
\hline Máster Educación Intercultural & 10 & 3 & 13 & 2,1 \\
\hline $\begin{array}{l}\text { Máster ESO, Bachillerato y } \\
\text { Formación Profesional }\end{array}$ & 108 & 24 & 132 & 21,5 \\
\hline No contesta & 15 & 12 & 27 & 4,4 \\
\hline
\end{tabular}

El cuestionario está compuesto por dos escalas y un test que se ha elaborado ad hoc con una función descriptiva, aunque en este documento exclusivamente se describe los resultados del test sobre conocimientos en relación al VIH/SIDA. El cuestionario completo consta de las siguientes partes que en total corresponden a 52 ítems:

- Datos sociodemográficos e información relevante sobre el encuestado: edad, sexo, titulación, curso, así como el interés hacia la temática planteada (item 1) y la fuente principal de información (ítem 2).

- Prueba tipo test sobre el VIH/SIDA (ítem 3-15). El objetivo de esta prueba es valorar el grado de conocimientos de los encuestados. Se ha procurado realizar preguntas con diferentes grados de dificultad para poder diferenciar entre aquellos sujetos que tienen gran cantidad y calidad de conocimientos y los que carecen de ellos.

- Escala categorizada sobre el campo de la representación social de los sujetos sobre el VIH/SIDA, cuyas preguntas intentarán abordar las creencias, percepciones y/o juicios de valor sobre este hecho (Ítem 16-38).

- Escala categorizada sobre las actitudes ante el VIH/SIDA de los estudiantes encuestados (Ítem 39-52).

El proceso de validación partió de la revisión de literatura (INJUVE, 2002; FIPSE, 2003; Moreno, et al., 2008; Morón-Marchena, et al., 2011) que concluyó con un listado de indicadores que fue sometida al juicio de 6 expertos. Tras el análisis se procede a la realización de una prueba piloto con una muestra de 36 sujetos. El análisis del Alfa del Cronbach en la prueba piloto permitió eliminar aquellos ítems que correlacionan poco con el conjunto y que, en consecuencia, permiten elevar el valor de Alfa. Asimismo, se eliminan aquellos ítems que hacen que aumente considerablemente la media de la escala (difíciles) y aquellos otros que provocan una disminución notable de la media (fáciles).

Para el análisis de la fiabilidad se aplica el Alfa de Cronbach, obteniendo un índice de 0,643 para 13 elementos y aplicado a 612 observaciones válidas. Teniendo 
Revista de Humanidades, 37 (2019).p. 127-150. ISSN 1130-5029

en cuenta que se trata de un test de acierto-error se puede considerar un valor aceptable. Asimismo, en el análisis de la consistencia interna, casi la totalidad de los indicadores tienen valores de correlación elemento-total corregida superiores a 0,171.

Tabla 5. Indicadores de consistencia interna del test Conocimientos

\begin{tabular}{lcccc}
\hline & $\begin{array}{c}\text { Media de la } \\
\text { escala si se } \\
\text { elimina el } \\
\text { elemento }\end{array}$ & $\begin{array}{c}\text { Varianza de } \\
\text { la escala si } \\
\text { se elimina el } \\
\text { elemento }\end{array}$ & $\begin{array}{c}\text { Correlación } \\
\text { elemento-total } \\
\text { corregida }\end{array}$ & $\begin{array}{c}\text { Alfa de } \\
\text { Cronbach si } \\
\text { se elimina el } \\
\text { elemento }\end{array}$ \\
\hline Términos_VIH_SIDA & 4,97 & 6,285 &, 000 &, 647 \\
\hline Persona_seropositiva & 4,31 & 5,373 &, 312 &, 618 \\
\hline Personas_VIH & 4,43 & 5,424 &, 264 &, 627 \\
\hline Paises_pocos_recursos & 4,59 & 5,217 &, 376 &, 606 \\
\hline Contagio_VIH_SIDA & 4,42 & 5,121 &, 406 &, 599 \\
\hline Felación & 4,50 & 5,311 &, 315 &, 617 \\
\hline Transmisión_horizontal & 4,65 & 5,406 &, 305 &, 619 \\
\hline Transmisión_vertical & 4,59 & 5,145 &, 411 &, 699 \\
\hline Enfermo_SIDA & 4,85 & 5,890 &, 182 &, 637 \\
\hline Carga_viral & 4,74 & 5,764 &, 171 &, 640 \\
\hline Riesgo_sexual & 4,81 & 5,858 &, 167 &, 611 \\
\hline Prueba_VIH & 4,44 & 5,248 &, 344 &, 642 \\
\hline Relaciones_sexuales & 4,33 & 5,652 &, 175 & \\
\hline
\end{tabular}

Tras el análisis de la fiabilidad se procede a la normalización de la escala, diferenciando percentiles de 10 en 10. En este sentido, se obtiene una media de 4,969, siendo la media 5 y la moda 5 . En términos generales, el percentil 30 se sitúa en 4, el percentil 60 se sitúa en 6 y el percentil 90 en 8 como se observa en la siguiente tabla:

Tabla 6. Descriptivos y percentiles del test Conocimientos

\begin{tabular}{|c|c|c|}
\hline \multirow{2}{*}{$\mathrm{N}$} & Válidos & 612 \\
\hline & Perdidos & 1 \\
\hline Media & & 49,690 \\
\hline Mediana & & 50,000 \\
\hline Moda & & 5,00 \\
\hline Desv. típ. & & 250,708 \\
\hline \multirow{10}{*}{ Percentiles } & 10 & 20,000 \\
\hline & 20 & 30,000 \\
\hline & 30 & 40,000 \\
\hline & 40 & 40,000 \\
\hline & 50 & 50,000 \\
\hline & 60 & 60,000 \\
\hline & 70 & 60,000 \\
\hline & 80 & 70,000 \\
\hline & 90 & 80,000 \\
\hline & 100 & 110,000 \\
\hline
\end{tabular}


La Prevención del VIH/SIDA... - C. M.A.Cejudo y C.Corchuelo

Tabla 7. Frecuencias y porcentajes del test Conocimientos

\begin{tabular}{|c|c|c|c|c|c|}
\hline & & Frecuencia & Porcentaje & Porcentaje válido & $\begin{array}{l}\text { Porcentaje } \\
\text { acumulado }\end{array}$ \\
\hline \multirow{13}{*}{ Válidos } & , 00 & 21 & 3,4 & 3,4 & 3,4 \\
\hline & 1,00 & 36 & 5,9 & 5,9 & 9,3 \\
\hline & 2,00 & 50 & 8,2 & 8,2 & 17,5 \\
\hline & 3,00 & 74 & 12,1 & 12,1 & 29,6 \\
\hline & 4,00 & 86 & 14,0 & 14,1 & 43,6 \\
\hline & 5,00 & 89 & 14,5 & 14,5 & 58,2 \\
\hline & 6,00 & 80 & 13,1 & 13,1 & 71,2 \\
\hline & 7,00 & 71 & 11,6 & 11,6 & 82,8 \\
\hline & 8,00 & 53 & 8,6 & 8,7 & 91,5 \\
\hline & 9,00 & 32 & 5,2 & 5,2 & 96,7 \\
\hline & 10,00 & 15 & 2,4 & 2,5 & 99,2 \\
\hline & 11,00 & 5 & 8 & 8 & 100,0 \\
\hline & Total & 612 & 99,8 & 100,0 & \\
\hline Perdidos & Sistema & 1 & ,2 & & \\
\hline Total & & 613 & 100,0 & & \\
\hline
\end{tabular}

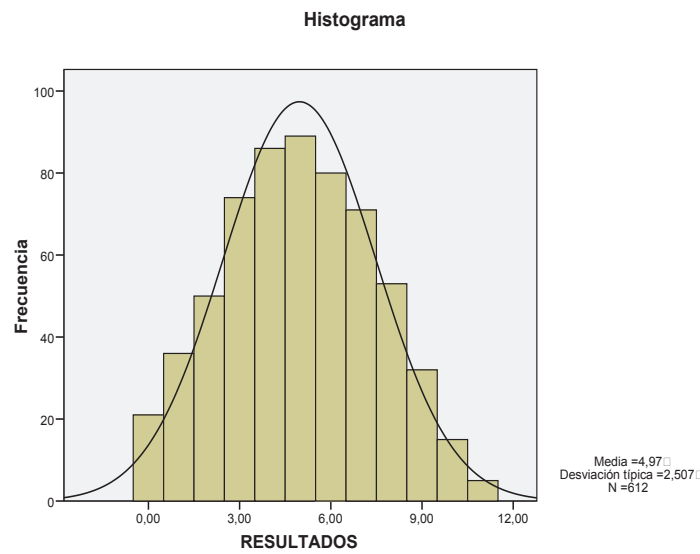

Gráfico 1. Histograma del Test Conocimientos

Se elaboró un calendario de administración del cuestionario una vez confirmado con los profesores que habían accedido voluntariamente a dejar un tiempo lectivo de clase para poder llevar a cabo la encuesta. Una vez entrado en el aula, se informaba a los encuestados sobre los aspectos abordados en el cuestionario, la relevancia e importancia de la encuesta, su carácter voluntario y las garantías de la confidencialidad de los datos. 


\section{RESULTADOS}

El primer ítem del cuestionario pretende conocer si los encuestados muestran interés por la temática que aborda el instrumento. En la tabla siguiente se puede apreciar que la opción c («Todo lo relacionado con el VIH/SIDA me interesa mucho») es la más predominante, con un $67,9 \%$ de las respuestas a favor. Este dato es significativo y favorable porque si los sujetos tienen interés al respecto, se puede prever que tengan una alta motivación en futuras actividades programadas.

Tabla 8. Interés de los sujetos por el VIH/SIDA

\begin{tabular}{|c|c|c|c|c|c|}
\hline & & Frecuencia & Porcentaje & Porcentaje válido & $\begin{array}{l}\text { Porcentaje } \\
\text { acumulado }\end{array}$ \\
\hline \multirow{6}{*}{ Válidos } & 0 & 1 & ,2 & ,2 &, 2 \\
\hline & Nada & 7 & 1,1 & 1,1 & 1,3 \\
\hline & Algo & 184 & 30,0 & 30,0 & 31,3 \\
\hline & Mucho & 416 & 67,9 & 67,9 & 99,2 \\
\hline & N.S./N.C & 5 & ,8 & ,8 & 100,0 \\
\hline & Total & 613 & 100,0 & 100,0 & \\
\hline
\end{tabular}

E1 70\% de las mujeres encuestadas les interesa mucho todos aquellos aspectos relacionados con el VIH/SIDA. Sin embargo, el porcentaje de hombres que tiene un interés alto con respecto a esta temática es inferior al del grupo de mujeres encuestadas $(52 \%)$.

Tabla 9. Contingencia entre el sexo y el interés de los sujetos sobre el VIH/SIDA

\begin{tabular}{|c|c|c|c|c|c|c|c|c|}
\hline & & & \multicolumn{5}{|c|}{ Interés_VIH_SIDA. } & \multirow[t]{2}{*}{ Total } \\
\hline & & & 0 & Nada & Algo & Mucho & N.S./N.C. & \\
\hline \multirow[t]{6}{*}{ Sexo } & 0 & Recuento & 1 & 0 & 0 & 5 & 0 & 6 \\
\hline & & $\begin{array}{l}\text { \% dentro de } \\
\text { Sexo }\end{array}$ & $16,7 \%$ & ,0\% &, $0 \%$ & $83,3 \%$ & ,0\% & $100,0 \%$ \\
\hline & Mujer & Recuento & 0 & 3 & 137 & 353 & 3 & 496 \\
\hline & & $\begin{array}{l}\text { \% dentro de } \\
\text { Sexo }\end{array}$ & ,0\% &, $6 \%$ & $27,6 \%$ & $71,2 \%$ & ,6\% & $100,0 \%$ \\
\hline & Hombre & Recuento & 0 & 4 & 47 & 58 & 2 & 111 \\
\hline & & $\begin{array}{l}\text { \% dentro de } \\
\text { Sexo }\end{array}$ &, $0 \%$ & $3,6 \%$ & $42,3 \%$ & $52,3 \%$ & $1,8 \%$ & $100,0 \%$ \\
\hline \multirow[t]{2}{*}{ Total } & & Recuento & 1 & 7 & 184 & 416 & 5 & 613 \\
\hline & & $\begin{array}{l}\% \text { dentro de } \\
\text { Sexo }\end{array}$ &, $2 \%$ & $1,1 \%$ & $30,0 \%$ & $67,9 \%$ & ,8\% & $100,0 \%$ \\
\hline
\end{tabular}


La Prevención del VIH/SIDA... - C. M.A.Cejudo y C.Corchuelo

Tabla 10. Pruebas Chi-cuadrado. Sexo e interés de los sujetos sobre VIH/SIDA

\begin{tabular}{llll}
\hline & Valor & Gl & $\begin{array}{l}\text { Sig. asintótica } \\
\text { (bilateral) }\end{array}$ \\
\hline Chi-cuadrado de Pearson & $123,385^{\text {a }}$ & 8 &, 000 \\
\hline Razón de verosimilitudes & 30,879 & 8 &, 000 \\
\hline Asociación lineal por lineal & 10,212 & 1 &, 001 \\
\hline N de casos válidos & 613 & & \\
\hline $\begin{array}{l}\text { a. } 10 \text { casillas (66,7\%) tienen una frecuencia esperada inferior a 5. La frecuencia } \\
\text { mínima esperada es }, 01 .\end{array}$ \\
\hline
\end{tabular}

Con respecto a la fuente que los sujetos expresan han obtenido la información que poseen en relación al VIH/SIDA, la opción más seleccionada ha sido la $\mathrm{C}$ (Charlas informativas en una institución escolar). Esta respuesta ha sido elegida por 263 alumnos/as (42\%). El segundo lugar corresponde a la opción A que se refiere a información recibida por «TV y/o documentales» con el 35\% (215 alumnos/as).

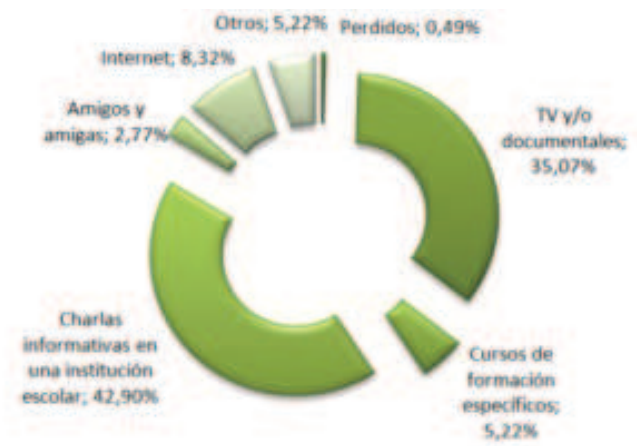

Gráfico 2. Fuente principal de la información que tienen los sujetos sobre el VIH/SIDA

La primera pregunta del test conocimiento -y tercera en el cuestionario- indaga si el alumnado diferencia entre la infección por VIH y la enfermedad del SIDA. La respuesta más seleccionada ha sido la opción correcta: «El SIDA es la fase más grave de la infección por VIH». Sin embargo, el porcentaje de estudiantes que ha seleccionado esta opción es de solo 36,9\%. En contraposición, el 63,1\% elige una respuesta incorrecta o no la contesta. 


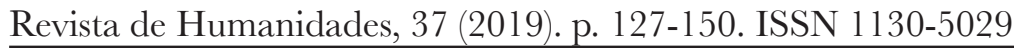

Tabla 11. Los términos VIH y SIDA

\begin{tabular}{|c|c|c|c|c|c|}
\hline \multicolumn{6}{|c|}{ Los términos VIH y SIDA } \\
\hline & & Frecuencia & Porcentaje & $\begin{array}{c}\text { Porcentaje } \\
\text { válido }\end{array}$ & $\begin{array}{l}\text { Porcentaje } \\
\text { acumulado }\end{array}$ \\
\hline \multirow[t]{6}{*}{ Válidos } & 0 & 6 & 1,0 & 1,0 & 1,0 \\
\hline & Significan lo mismo & 187 & 30,5 & 30,5 & 31,5 \\
\hline & $\begin{array}{l}\text { EI SIDA es la fase más } \\
\text { grave de la infección } \\
\text { por VIH }\end{array}$ & 226 & 36,9 & 36,9 & 68,4 \\
\hline & $\begin{array}{l}\text { El VIH es la fase más } \\
\text { grave de la infección } \\
\text { por SIDA }\end{array}$ & 33 & 5,4 & 5,4 & 73,7 \\
\hline & N.S. o N.C. & 161 & 26,3 & 26,3 & 100,0 \\
\hline & Total & 613 & 100,0 & 100,0 & \\
\hline
\end{tabular}

El ítem 4 de nuestro cuestionario pregunta sobre el significado de la condición de seropositividad. En esta cuestión, los datos son mucho más favorables (el 65\% de los encuestados saben que una persona es seropositiva desde el momento en el que se infecta por VIH). Ya se preveía este hecho, ya que en el cuestionario piloto se pudo observar que se trataba de un ítem de dificultad baja.

Tabla 12. Persona seropositiva

\begin{tabular}{|c|c|c|c|c|c|}
\hline \multicolumn{6}{|c|}{ «Una persona es seropositiva cuando...» } \\
\hline & & Frecuencia & Porcentaje & $\begin{array}{l}\text { Porcentaje } \\
\text { válido }\end{array}$ & $\begin{array}{l}\text { Porcentaje } \\
\text { acumulado }\end{array}$ \\
\hline \multirow[t]{6}{*}{ Válidos } & 0 & 6 & 1,0 & 1,0 & 1,0 \\
\hline & $\begin{array}{l}\text { Si en la prueba del } \\
\text { VIH los resultados no } \\
\text { detectan el VIH }\end{array}$ & 27 & 4,4 & 4,4 & 5,4 \\
\hline & $\begin{array}{l}\text { Desde el momento } \\
\text { que se infecta por VIH }\end{array}$ & 404 & 65,9 & 65,9 & 71,3 \\
\hline & $\begin{array}{l}\text { Si se encuentra en la } \\
\text { fase más grave de la } \\
\text { infección }\end{array}$ & 26 & 4,2 & 4,2 & 75,5 \\
\hline & N.S. o N.C. & 150 & 24,5 & 24,5 & 100,0 \\
\hline & Total & 613 & 100,0 & 100,0 & \\
\hline
\end{tabular}

Sin perder de vista los anteriores resultados, se analiza a continuación la relación de cada una de las repuestas con la edad de los sujetos. El alumnado que mayor puntuación ha obtenido- ya que ha seleccionado un número mayor de estudiantes la pregunta correcta- corresponde al intervalo de edad «mayor de 25 años» con un $74,8 \%$, mientras que los menores o iguales a 21 años de edad, tienen el menor porcentaje de aciertos $(59,8 \%)$. El alumnado de mayor edad tiene una información más exhaustiva sobre la temática que abordamos. 
La Prevención del VIH/SIDA... - C. M.A.Cejudo y C.Corchuelo

Tabla 13. Edad-Ítem 4

\begin{tabular}{|c|c|c|c|c|c|c|c|}
\hline \multicolumn{8}{|c|}{ Contingencia. Edad- ítem 4} \\
\hline & & \multicolumn{5}{|c|}{ Persona_seropositiva. } & \multirow[b]{2}{*}{ Total } \\
\hline & & 0 & $\begin{array}{l}\text { Si en la prueba } \\
\text { del VIH los } \\
\text { resultados no } \\
\text { detectan el VIH }\end{array}$ & $\begin{array}{l}\text { Desde el } \\
\text { momento } \\
\text { que se } \\
\text { infecta por } \\
\text { VIH }\end{array}$ & $\begin{array}{c}\text { Si se } \\
\text { encuentra en } \\
\text { la fase más } \\
\text { grave de la } \\
\text { infección }\end{array}$ & $\begin{array}{l}\text { N.S. o } \\
\text { N.C. }\end{array}$ & \\
\hline \multirow{2}{*}{$\begin{array}{l}21 \text { años } \\
\text { o menos }\end{array}$} & Recuento & 1 & 7 & 101 & 9 & 51 & 169 \\
\hline & $\begin{array}{l}\text { \% dentro de } \\
\text { Edad_test }\end{array}$ & ,6\% & $4,1 \%$ & $59,8 \%$ & $5,3 \%$ & $30,2 \%$ & $100,0 \%$ \\
\hline \multirow{2}{*}{$\begin{array}{l}\text { Entre } 21 \\
\text { hasta } 23\end{array}$} & Recuento & 1 & 7 & 116 & 5 & 34 & 163 \\
\hline & $\begin{array}{l}\% \text { dentro de } \\
\text { Edad_test }\end{array}$ & ,6\% & $4,3 \%$ & $71,2 \%$ & $3,1 \%$ & $20,9 \%$ & $100,0 \%$ \\
\hline \multirow{2}{*}{$\begin{array}{l}\text { Entre } 23 \\
\text { hasta } 25\end{array}$} & Recuento & 0 & 6 & 85 & 7 & 40 & 138 \\
\hline & $\begin{array}{l}\text { \% dentro de } \\
\text { Edad_test }\end{array}$ &, $0 \%$ & $4,3 \%$ & $61,6 \%$ & $5,1 \%$ & $29,0 \%$ & $100,0 \%$ \\
\hline \multirow{4}{*}{$\begin{array}{l}\text { Mayor } \\
\text { de } 25 \\
\text { años }\end{array}$} & Recuento & 1 & 6 & 89 & 3 & 20 & 119 \\
\hline & $\begin{array}{l}\text { \% dentro de } \\
\text { Edad_test }\end{array}$ &, $8 \%$ & $5,0 \%$ & $74,8 \%$ & $2,5 \%$ & $16,8 \%$ & $100,0 \%$ \\
\hline & Recuento & 3 & 26 & 391 & 24 & 145 & 589 \\
\hline & $\begin{array}{l}\text { \% dentro de } \\
\text { Edad_test }\end{array}$ &, $5 \%$ & $4,4 \%$ & $66,4 \%$ & $4,1 \%$ & $24,6 \%$ & $100,0 \%$ \\
\hline
\end{tabular}

Tabla 14. Pruebas de chi-cuadrado entre la Edad y el significado de Persona Seropositiva

\begin{tabular}{lccc}
\hline \multicolumn{1}{c}{ Prueba Chi-cuadrado } & Valor & Gl & $\begin{array}{c}\text { Sig. asintótica } \\
\text { (bilateral) }\end{array}$ \\
\hline Chi-cuadrado de Pearson & $13,751^{\text {a }}$ & 12 &, 317 \\
\hline Razón de verosimilitudes & 14,683 & 12 &, 259 \\
\hline Asociación lineal por lineal & 4,100 & 1 &, 043 \\
\hline N de casos válidos & 589 & & \\
\hline $\begin{array}{l}\text { a. } 5 \text { casillas (25,0\%) tienen una frecuencia esperada inferior a 5. La frecuencia mínima } \\
\text { esperada es ,61. }\end{array}$ & & \\
\hline
\end{tabular}

En la pregunta 5 del cuestionario se ha preguntado «si todas las personas con VIH, desarrollarán la enfermedad del SIDA». Los datos son relativamente positivos ya que nos encontramos con un $54 \%$ de personas que piensan que no tienen por qué desarrollar el SIDA. Sin embargo, aún muchas personas -el 18\% de los encuestados que forman la muestra- siguen pensando que todas las personas que tienen $\mathrm{VIH}$, tienen SIDA. 


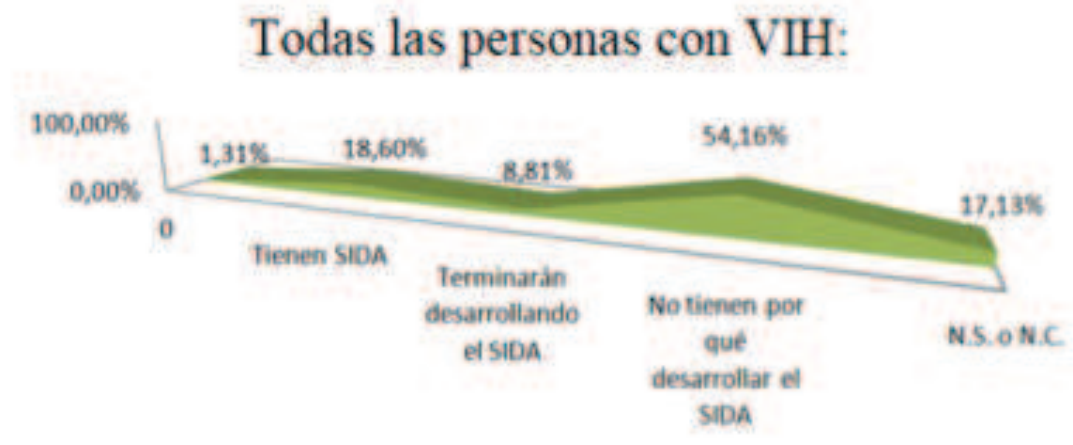

Gráfico 3. Personas VIH y enfermedad del SIDA

En relación a la cuestión planteada, «el VIH se puede contagiar a través de...», la opción más seleccionada ha sido la correcta. El 54,8\% de los sujetos, discrimina que las lágrimas, sudor, saliva, tos o estornudos, no son vías de transmisión, habiendo seleccionado la opción correcta en esta pregunta, la leche materna. El 45,2\% restante, no tiene una información real o completa sobre las vías de transmisión, pues han elegido o una opción no correcta o se han declinado por última opción («No sé, no contesto»).

Tabla 15. Contagio del VIH

\begin{tabular}{llcccc}
\hline El VIH se puede contagiar a través de: & & & & \\
\hline \multirow{3}{*}{ Válidos } & Frecuencia & Porcentaje & $\begin{array}{c}\text { Porcentaje } \\
\text { válido }\end{array}$ & $\begin{array}{c}\text { Porcentaje } \\
\text { acumulado }\end{array}$ \\
\cline { 2 - 6 } & Valores perdidos & 9 & 1,5 & 1,5 & 1,5 \\
\cline { 2 - 6 } & lágrimas, sudor, y saliva & 104 & 17,0 & 17,0 & 18,4 \\
\cline { 2 - 6 } & tos y estornudos & 6 & 1,0 & 1,0 & 19,4 \\
\cline { 2 - 6 } & leche materna & 336 & 54,8 & 54,8 & 74,2 \\
\cline { 2 - 6 } & N.S. o N.C. & 158 & 25,8 & 25,8 & 100,0 \\
\cline { 2 - 6 } & Total & 613 & 100,0 & 100,0 & \\
\hline
\end{tabular}

En este ítem hemos preguntado por una de las prácticas orales, concretamente la felación, para poder saber si los encuestados manejan o no esta información como una de las posibles vías de transmisión. Casi la mitad de los encuestados $(47,3 \%)$, eligen la opción correcta («el riesgo mayor se produce para quien recibe la eyaculación en la boca»). El 52,7\% restante o ha elegido una opción incorrecta, o no sabe o no contesta al respecto. Por lo que se puede decir que únicamente la mitad de los sujetos de nuestra muestra tienen una información verídica sobre esta práctica sexual y su probabilidad de contagio en una hipotética transmisión. 
La Prevención del VIH/SIDA... - C. M.A.Cejudo y G.Corchuelo

Tabla 16. Felación y transmisión del VIH

\begin{tabular}{llcccc}
\hline Cuando se practica una felación con una persona con VIH/SIDA: & & & \\
\hline & Frecuencia & Porcentaje & $\begin{array}{c}\text { Porcentaje } \\
\text { válido }\end{array}$ & $\begin{array}{c}\text { Porcentaje } \\
\text { acumulado }\end{array}$ \\
\hline Válidos & 12 & 2,0 & 2,0 & 2,0 \\
\hline & $\begin{array}{l}\text {-Valores perdidos } \\
\begin{array}{l}\text {-No hay riesgo de contagio } \\
\text { porque no hay penetración }\end{array}\end{array}$ & 53 & 8,6 & 8,6 & 10,6 \\
\hline $\begin{array}{l}\text {-El riesgo mayor se produce para } \\
\text { quien recibe la eyaculación en } \\
\text { la boca }\end{array}$ & 290 & 47,3 & 47,3 & 57,9 \\
$\begin{array}{l}\text { El riesgo mayor es para el sujeto } \\
\text { al que le están practicando la } \\
\text { felación }\end{array}$ & 113 & 18,4 & 18,4 & 76,3 \\
\hline $\begin{array}{l}\text {-N.S. o N.C. } \\
\text { Total }\end{array}$ & 145 & 23,7 & 23,7 & 100,0 \\
\hline
\end{tabular}

En relación a la cuestión que planteamos sobre el significado de transmisión vertical -sabiendo por la prueba piloto que tenía una dificultad media - el 37\% elige la respuesta correcta («de madres a hijos/as»). No obstante, el $41 \%$ de los encuestados no saben el significado de transmisión vertical.

Cuando hablamos de transmisión vertical, nos referimos al contagio que se produce:

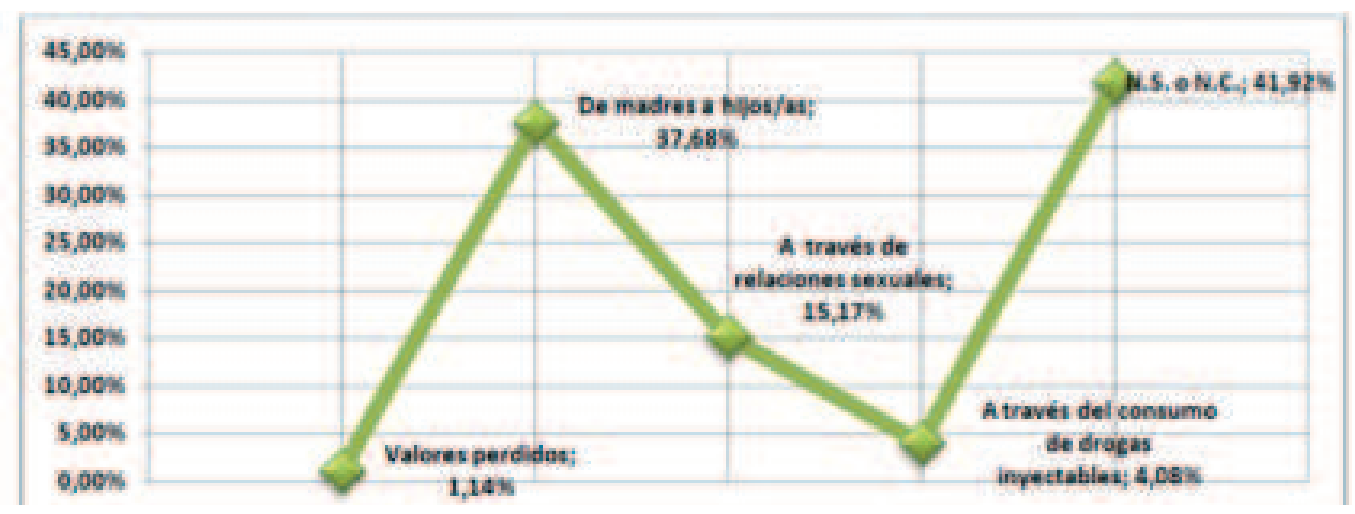

Gráfico 4. Transmisión vertical

En la pregunta 14 del cuestionario se consulta sobre dónde se puede realizar la prueba del VIH. Un gran número de encuestados $(53,2 \%)$, eligen la opción en 
Revista de Humanidades, 37 (2019).p. 127-150. ISSN 1130-5029

«cualquier centro de salud», no obstante, el resto tiene una información errónea o inexacta de las instituciones sanitarias donde se puede realizar dicha prueba.

Tabla 17. Prueba VIH

\begin{tabular}{|c|c|c|c|c|c|}
\hline \multicolumn{6}{|c|}{ La prueba del VIH se llevan a cabo: } \\
\hline & & Frecuencia & Porcentaje & $\begin{array}{l}\text { Porcentaje } \\
\text { válido }\end{array}$ & $\begin{array}{l}\text { Porcentaje } \\
\text { acumulado }\end{array}$ \\
\hline \multirow[t]{6}{*}{ Válidos } & Perdidos & 13 & 2,1 & 2,1 & 2,1 \\
\hline & $\begin{array}{l}\text { Exclusivamente en } \\
\text { laboratorios privados }\end{array}$ & 26 & 4,2 & 4,2 & 6,4 \\
\hline & $\begin{array}{l}\text { En cualquier centro de salud } \\
\text { público aunque la prueba no } \\
\text { es gratuita }\end{array}$ & 75 & 12,2 & 12,2 & 18,6 \\
\hline & En cualquier centro de salud & 326 & 53,2 & 53,2 & 71,8 \\
\hline & N.S. o N.C. & 173 & 28,2 & 28,2 & 100,0 \\
\hline & Total & 613 & 100,0 & 100,0 & \\
\hline
\end{tabular}

Como ha pasado en gran parte de las respuestas obtenidas en el tipo test, los mayores de 25 años son los que han obtenido un porcentaje superior en la respuesta correcta y el menor en la respuesta «No sé, no contesto».

Tabla 18. Prueba VIH-Edad

\begin{tabular}{|c|c|c|c|c|c|c|c|}
\hline \multicolumn{8}{|c|}{ Contingencia. Edad-Prueba del VIH. } \\
\hline & & Perdidos & $\begin{array}{c}\text { exclusivamente } \\
\text { en laboratorios } \\
\text { privados }\end{array}$ & $\begin{array}{c}\text { en cualquier } \\
\text { centro de salud } \\
\text { público aunque } \\
\text { la prueba no es } \\
\text { gratuita }\end{array}$ & $\begin{array}{l}\text { en } \\
\text { cualquier } \\
\text { centro de } \\
\text { salud }\end{array}$ & $\begin{array}{l}\text { N.S. } 0 \\
\text { N.C. }\end{array}$ & Total \\
\hline \multirow{2}{*}{$\begin{array}{l}21 \text { años } \\
\text { o menos }\end{array}$} & Recuento & 5 & 8 & 22 & 85 & 49 & 169 \\
\hline & $\begin{array}{l}\% \text { dentro de } \\
\text { Edad_test }\end{array}$ & $3,0 \%$ & $4,7 \%$ & $13,0 \%$ & $50,3 \%$ & $29,0 \%$ & $100,0 \%$ \\
\hline \multirow{2}{*}{$\begin{array}{l}\text { Entre } 21 \\
\text { hasta } 23\end{array}$} & Recuento & 2 & 4 & 20 & 85 & 52 & 163 \\
\hline & $\begin{array}{l}\% \text { dentro de } \\
\text { Edad_test }\end{array}$ & $1,2 \%$ & $2,5 \%$ & $12,3 \%$ & $52,1 \%$ & $31,9 \%$ & $100,0 \%$ \\
\hline \multirow{2}{*}{$\begin{array}{l}\text { Entre } 23 \\
\text { hasta } 25\end{array}$} & Recuento & 2 & 8 & 15 & 67 & 46 & 138 \\
\hline & $\begin{array}{l}\text { \% dentro de } \\
\text { Edad_test }\end{array}$ & $1,4 \%$ & $5,8 \%$ & $10,9 \%$ & $48,6 \%$ & $33,3 \%$ & $100,0 \%$ \\
\hline \multirow{4}{*}{$\begin{array}{l}\text { Mayor de } \\
25 \text { años }\end{array}$} & Recuento & 2 & 5 & 14 & 76 & 22 & 119 \\
\hline & $\begin{array}{l}\text { \% dentro de } \\
\text { Edad_test }\end{array}$ & $1,7 \%$ & $4,2 \%$ & $11,8 \%$ & $63,9 \%$ & $18,5 \%$ & $100,0 \%$ \\
\hline & Recuento & 11 & 25 & 71 & 313 & 169 & 589 \\
\hline & $\begin{array}{l}\% \text { dentro de } \\
\text { Edad_test }\end{array}$ & $1,9 \%$ & $4,2 \%$ & $12,1 \%$ & $53,1 \%$ & $28,7 \%$ & $100,0 \%$ \\
\hline
\end{tabular}


La Prevención del VIH/SIDA... - C. M.A.Cejudo y C.Corchuelo

Tabla 19. Chi-cuadrado de Pearson entre la Edad y la Prueba VIH

\begin{tabular}{lccc}
\hline & Prueba Chi-cuadrado & & \\
\hline & Valor & Gl & $\begin{array}{c}\text { Sig. asintótica } \\
\text { (bilateral) }\end{array}$ \\
\hline Chi-cuadrado de Pearson & $13,359^{\mathrm{a}}$ & 12 &, 343 \\
\hline Razón de verosimilitudes & 13,817 & 12 &, 313 \\
\hline Asociación lineal por lineal &, 129 & 1 &, 719 \\
\hline N de casos válidos & 589 & & \\
\hline a. 4 casillas (20,0\%) tienen una frecuencia esperada inferior a 5. La frecuencia mínima esperada es \\
\end{tabular}

En este sentido, los datos proporcionados por los estudiantes universitarios de titulaciones educativas de la Universidad de Huelva, confirman que hay un gran porcentaje que, a pesar de haber recibido formación específica en instituciones escolares, sigue sin conocer aspectos básicos del VIH/SIDA. Por ello, se estima que ofrecer información más exhaustiva, concreta e insistente en edades tempranas, puede ayudar a prevenir nuevas infecciones. La información precedería a las primeras relaciones sexuales, y de ese modo los preadolescentes y adolescentes adquirirían las competencias necesarias para evitar riesgos innecesarios, a veces con fatales consecuencias.

\section{A MODO DE GONGLUSIÓN: ANTICIPAR Y POTENGIAR LA FORMAGIÓN PREVENTIVA}

Los resultados obtenidos en nuestra investigación nos aportan pistas sobre cómo enfocar la formación del alumnado universitario de titulaciones educativas en relación con el VIH/SIDA. Desafortunadamente, la formación específica en la prevención de enfermedades de transmisión sexual, como el VIH, se suele desarrollar, generalmente, después de las primeras relaciones sexuales. Para una prevención realmente eficaz, la formación en estos aspectos debe llegar antes y continuar en el tiempo, ofreciendo más y mejor información que fomente conductas y hábitos sexuales saludables.

Para lograr el éxito de este tipo de iniciativas, deberíamos emplear diferentes metodologías pedagógicas adecuadas. Ofrecer únicamente información no produce los resultados deseados. La mayoría de los estudiantes ha recibido información en diversos formatos durante su escolarización y, sin embargo, la mitad de ellos, siendo ya universitarios, muestra carencias importantes. Deberían promoverse actividades basadas en el diálogo y el debate, con el objetivo de contribuir a la desaparición de conocimientos erróneos, representaciones sociales negativas y actitudes que discriminan y aíslan a quienes padecen el VIH/SIDA. Es fundamental que los jóvenes se consideren protagonistas en las actividades de prevención dirigidas a 


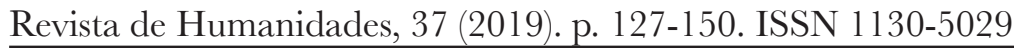

ellos mismos y que, en consecuencia, se sientan más implicados y motivados, de modo que asuman un mayor compromiso consigo y con los demás.

Unos jóvenes, que, en función de su edad, van asimilando una serie de conceptos acerca del VIH/SIDA y que es algo que no se encuentra ligado a una titulación u otra, siempre que nos refiramos a titulaciones de índole educativa, sino más bien es algo relacionado con su edad y madurez biológica y psicológica, es decir, va a depender de los conocimientos, las actitudes y las representaciones que tenga esa persona ante el virus del VIH.

Es conveniente también que toda la comunidad educativa participe en la prevención de hábitos saludables en general y en el caso del VIH/SIDA en particular. Una comunidad que debe de ejercer de colaborador para que la juventud se forme en estos ámbitos. Tanto es así, que la familia debe ser el prisma donde los jóvenes encaucen su camino en esta prevención y los profesionales, más aún si cabe, deben actuar como guías para facilitar el conocimiento sobre estos temas en el alumnado. Para ello, es imprescindible crear futuros docentes formados en prevención y en didáctica, capaces de mostrar una serie de recursos docentes para que el alumnado sea capaz de poder asimilar todo el contenido propuesto por el profesorado, y sea a su vez, capaz de trasmitirlo de la misma manera a generaciones posteriores.

Se trata, en definitiva, de construir un entorno favorable en las universidades para sensibilizar a los jóvenes en la necesidad de proteger su salud, con la implementación de programas formativos que los persuadan de la importancia de practicar sexo seguro, de la necesidad de las pruebas diagnósticas si se han realizado prácticas de riesgo y sobre todo, de la urgencia de combatir la estigmatización que sufren las personas que sufren las personas con VIH/SIDA.

\section{REFERENCIAS BIBLIOGRÁFICAS}

Aramendi, P., Buján, K. y Arburua, R. (2014). Educación para la Salud e intervención educativa en la Educación Secundaria Obligatoria. La percepción del alumnado. Revista Española de Pedagogía, 259, pp. 543-566.

Bilbao Ramírez, J.L., Crespo Camacho, E.J., De la Hoz Herrera, G.E. y Alcocer Olaciregui, A.E.. (2017). Nivel de conocimientos, tipo de actitud y prácticas de los estudiantes de medicina sobre el VIH/SIDA (Barranquilla, Colombia): estudio descriptivo. Archivos de Medicina (Col), vol. 17, núm. 1, enero-junio, 2017, pp. 54-63.

Burkhart, Gregor (2009). Creencias normativas en estrategias preventivas: una espada de doble filo. Efectos de la percepción de normas y normalidad en campañas informativas, programas escolares y medidas ambientales. Revista Española de Drogodependencias, 34(4), pp. 376-400. 
La Prevención del VIH/SIDA... - C. M.A.Cejudo y C.Corchuelo

Cassá, C., Ballester, R., Gil, M., Giménez, C., Gómez, S. (2009). Incremento de las prácticas de riesgo en los jóvenes: últimas tendencias en el uso del preservativo. Encuentro FIPSE sobre investigación de la perspectiva social del VIH/sida en San Sebastián: Fundación para la Investigación y la Prevención del Sida en España.

Cava, M. y Musitu, G. (2002). La convivencia en la escuela. Barcelona: Paidós.

Deblonde, J., de Koker, P., Hamers, F., Fontaine, J., Luchters, S., Temmerman, M. (2010). Barriers to HIV testing in Europe: A systematic review. The European Journal of Public Health, 20, pp. 422-432.

De Wit, J., Adam, P. (2008).To test or not to test: Psychosocial barriers to HIV testing in high-income countries. HIV Medicine, 9, pp. 20-22.

Domínguez Ramírez, L., Beltrán Delgado, Y., y Horta Sánchez, M. (2017). La preparación del docente para la promoción de la salud. Revista Márgenes, 5(2), pp. 26-37, abril-junio. Disponible en: http://revistas.uniss.edu.cu/index.php/margenes/ issue/view/614

Fernández, L.; Fernández, P. y Sábado, J. (2006). Modificación de actitudes ante el Sida en estudiantes de enfermería. Resultados de una experiencia pedagógica. Educación Médica 9(2).

Fundación para la Innovación y la Prospectiva en Salud en España (FIPSE) (2003). Informe Jóvenes, relaciones sexuales y riesgo de infección por VIH. Encuesta de Salud y Hábitos Sexuales. San Sebastián: Fundación para la Investigación y la Prevención del Sida en España. Disponible en: http://www.fipse.es/mixto/ biblioteca/00000096/00000191/827/20090328232325.pdf

Giménez, C., Ballester, R., Gil, M., Ruiz, E. y Cassá, C. (2009). ¿Qué resulta más eficaz en la prevención del VIH-Sida? Análisis diferencial de distintas estrategias de intervención para la promoción de la realización de las pruebas de anticuerpos de VIH en población joven. II Encuentro FIPSE sobre investigación social en VIH/SIDA. (pp. 214-223). San Sebastián: Fundación para la Investigación y la Prevención del Sida en España.

González, M. (2009). Intervención de la Universidad en la promoción de la salud de sus estudiantes. Innovación Educativa, 19, pp. 247-260. Editorial Universidad Santiago de Compostela.

Hoyos, J., De la Fuente, L., Fernández, S., Gutiérrez, J., Rosales, M., García, P., Ruiz, M., Belza, M. (2011). La oferta de la prueba rápida del VIH en la calle dentro del ámbito universitario: ¿una estrategia prioritaria? Gaceta Sanitaria, 26(2), pp. 131-137.

INJUVE, Instituto de la Juventud, (2002). Sondeo periódico de opinión y situación de la gente joven. Tercer trimester de 2002. Madrid. Instituto de la Juventud.

Mahajan, A., Sayles, J., Patel, V., Remien, R., Sawires, S., Ortiz, D., Szekeres, G., Coates, T. (2008). Stigma in the HIV/AIDS epidemic: A review of the literature and recommendations for the way forward. AIDS, 22 (Suppl. 2), pp. 67-79.

Martxueta, A., Etxeberría, J. (2014). Claves para atender a la diversidad afectivo sexual en el contexto educativo desde un enfoque global escolar. Revista Española de Orientación y Psicopedagogía, 25(3), pp. 121-128. 
Revista de Humanidades, 37 (2019). p. 127-150. ISSN 1130-5029

Meneses, C., Romo, N., Uroz, J., Gil, E., Markez, I., Giménez, S. y Vega, A. (2009). Adolescencia, consumo de drogas y comportamientos de riesgo: diferencias por sexo, etnicidad y áreas geográficas en España. Trastornos Adictivos. 11(1), pp. 51-63.

Meza-Leon J, Conislla Monterola J, Huarancca Gavilan L, Soto Meneses K. (2017). Nivel de conocimientos sobre infecciones de trasmisión sexual VIH/SIDA y sus medidas preventivas en estudiantes de Ciencias de la Salud de Ica. Rev méd panacea. Volumen 6 (3), pp. $104-110$.

Ministerio de Sanidad y Consumo. (2008). Plan Multisectorial frente a la infección por VIH y el SIDA. España. Disponible en: https://www.msssi.gob.es/ciudadanos/ enfLesiones/enfTransmisibles/sida/docs/PMS200812.pdf

Morales, A., Espada, J., Orgilés, M. (2016). Barreras hacia la prueba de detección del VIH en adolescentes en España. Psychosocial Intervention, 25(3), pp. 135-141.

Morán, L y Tarajano, A. (Noviembre de 2017). La educación sexual en la prevención de las ITS/VIH/sida en las Universidades. En XVII Congreso de la Sociedad Cubana de Enfermería. Congreso llevado a cabo en La Habana, Cuba.

Moreno, M., Rivera, F., Ramos, P., Jiménez, A., Muñoz, M., Sánchez, M., Granado, M. (2008). Las conductas relacionadas con la salud y el desarrollo de los adolescentes españoles. Resultados del estudio HBSC-2010 con chicos y chicas españoles de 11 a 18 años. Disponible en: http://www.mscbs.gob.es/profesionales/saludPublica/ prevPromocion/promocion/saludJovenes/estudioHBSC/docs/HBSC2010/ HBSC2010_Completo.pdf

Morón-Marchena, J.A., López-Noguero, F., Sanchiz, D. C., y Torres-Gordillo, J. J. (2011). SIDA: Conocimientos, actitudes y prácticas de riesgo en estudiantes y profesores en el CUR-Chontales de la Universidad Nacional Autónoma de Nicaragua-Managua. En La problemática del SIDA: una aproximación socioeducativa (pp. 35-86). Universidad Pablo de Olavide, de Sevilla.

Organización Mundial de la Salud (OMS) (2010). Escuelas Promotoras de Salud. Disponible en: http://www.paho.org/spanish/hpp/hpm/hec/hs_about.htm

ONUSIDA (2018). Hoja Informativa. Ginebra: Programa Conjunto de las Naciones Unidas sobre el VIH/SIDA. Disponible en: http://www.unaids.org/sites/default/ files/media_asset/UNAIDS_FactSheet_es.pdf

Osorio Leyva,A., ÁlvarezAguirre,A.,Hernández Rodríguez, V.M., SánchezPerales, M., \& Muñoz Alonso, L. D. R. (2017). Relación entre asertividad sexual y autoeficacia para prevenir el VIH/SIDA en jóvenes universitarios del área de la salud. RIDE. Revista Iberoamericana para la Investigación y el Desarrollo Educativo, 7(14), pp. 1-14.

Palomares, J., Cimarro, J., Cepero, M., Torres, B., Estévez, M., Martínez, R. (2014). Entre la teoría y la realidad: opiniones y creencias del profesorado sobre la transmisión y fomento de los hábitos saludables, a través del deporte y el juego, en la compleja relación escuela-familia. Revista de Currículum y Formación del Profesorado, 18(2), pp. 135-151.

Pérez, I. (2013). Conocimientos y prácticas de riesgo en VIH/SIDA. Una estrategia de Cooperación en Educación para el Desarrollo en Nicaragua (Tesis Doctoral). Sevilla: Universidad Pablo de Olavide. 
La Prevención del VIH/SIDA... - C. M.A.Cejudo y C.Corchuelo

Prost, A., Elford, J., Imrie, J., Petticrew, M., Hart, G. (2008). Social, behavioural, and intervention research among people of Sub-Saharan African origin living with HIV in the UK and Europe: Literature review and recommendations for intervention. AIDS and Behavior, 12, pp. 170-194.

Rojas-Murcia, C., Pastor, Y., Esteban, J. (2014). Ilusión de invulnerabilidad, estereotipos y percepción de control del SIDA en universitarios. Revista Iberoamericana de Psicología y Salud, 6(1), pp. 28-38.

Salamanca Ramos E, Romero González E. (2017). Comportamiento ante la transmisión del VIH/sida en adolescentes y jóvenes en universidades privadas de Villavicencio (Meta, Colombia). Investig. Enferm. Imagen Desarr., 19(2), pp. 53-67. http:// dx.doi.org/10.11144/Javeriana.ie19-2.ctva

Salvador, T. (2008). Informe: diagnóstico de situación sobre avances conseguidos, necesidades y retos en promoción y educación para la salud en la escuela en España. Madrid: Ministerio de Sanidad y Consumo. Disponible en: https://www.mscbs. gob.es/profesionales/saludPublica/prevPromocion/promocion/saludJovenes/docs/ DiagnosticoSituacionEscuela.pdf

Schenker, I. y Nyirenda, J. (2002). Prevención del VIH-Sida en las escuelas. Bogotá: Cooperativa Editorial de Magisterio.

Tello, M. (2009). Conocimientos y actitudes de estudiantes universitarios frente al VIH/ SIDA. II Encuentro FIPSE sobre investigación social en VIH/SIDA. San Sebastián: Fundación para la Investigación y la Prevención del SIDA en España.

UNAIDS, (2010). Getting to Zero: 2011-2015 Strategy. World Health Organization. Joint United Nations Programme on HIV/AIDS.

UNESCO (2012). Estrategia de la UNESCO sobre el VIH/SIDA. Organización de las Naciones Unidas para la Educación, la Ciencia y la Cultura. París, Francia. Disponible en: http://unesdoc.unesco.org/images/0019/001931/193118s.pdf

Vega, A., Aramendi, P., Buján, K., Garín, S. (2015). La educación para la salud en la ESO: Aportaciones de un estudio sobre el País Vasco. Educación XX1, 18(1), pp. 167-188. 